$\mathrm{D}$ ie älteren Ärzte werden sich noch an die späten 60er und frühen 70er Jahre des vergangenen Jahrhunderts erinnern können, als viele Patienten wegen unklarer Bewusstlosigkeit in neurologische Kliniken eingeliefert wurden und sich erst dort herausstellte, dass sie unterzuckert waren. Der damals neu eingeführte, sehr potente Sulfonylharnstoff Glibenclamid, anfangs auch in zu hohen Dosierungen gegeben, hatte nämlich in einem viel höheren Prozentsatz zu Hypoglykämien geführt als die alten Sulfonylharnstoffe der ersten Generation. Man sollte meinen, dass dies heute, insbesondere wo die Schnelldiagnostik durch Blutzucker-Teststreifen verfügbar ist, nicht mehr passiert. Ich kann mich jedoch an zwei Fälle aus der letzten Zeit erinnern, wo in Deutschland Patienten in der Nacht mit dem Notarztwagen in Aufnahmestationen gebracht wurden und unter der Annahme eines zerebralen Prozesses eine computertomographische Untersuchung durchgeführt wurde, ohne dass das Resultat einer Blutzucker-Testung vorgelegen hätte. Der erst nachher eingegangene Glukose-Messwert im "Routine-Labor" hatte dann bei 17 bzw. 25 $\mathrm{mg} / \mathrm{dl}$ gelegen. Aber nicht nur mit Glibenclamid, sondern auch mit den neueren Sulfonylharnstoff-Präparaten wie Glimepirid werden, insbesondere bei Niereninsuffizienz oder auch bei zu hoher Dosierung bzw. älteren Patienten, schwere und schwerste Hypoglykämien beobachtet, welche Fremdhilfe erforderlich machen (Holstein et al., 2003). Oft wird dem Notarzt nicht bekannt, dass der Patient einen Diabetes hat, es kommt aber auch in Altenheimen vor, dass verwirrte ältere Menschen Tabletten von Mitinsassen zu sich nehmen. Unterzuckerungen können in sehr seltenen Fällen auch bei Nicht-Diabetes-Patienten auftreten, wenn sie keine Medikamente zu sich nehmen: beim Vorliegen eines Insulinoms, einer insulinproduzierenden Inselzell-Geschwulst, bei anderen, großen Tumoren, oder in der Pädiatrie bei der - äußerst raren - Leucin-Überempfindlichkeit. Für den Notarzt muss also der Grundsatz gelten: bei einem bewusstlosen Patienten immer sofort den Blutzuckerspiegel mit Teststreifen prüfen!

Je besser, das heißt je normnäher ein Diabetes-Patient eingestellt ist, um so mehr Unterzuckerungsreaktionen wird er erleiden, dies gilt sowohl für Typ 1- als auch für Typ 2-Diabetes-Patienten. Patienten mit Typ 1-Diabetes neigen freilich besonders stark zu Hypoglykämien, da sie nicht insulinresistent wie sehr viele Typ 2Diabetes-Patienten sind. Gehäufte, schwere Hypoglykämien sind eine allgemein anerkannte Indikation zur stationären Einweisung. In besonderem Maße gilt dies für Sulfonylharnstoff-induzierte Hypoglykämien. Diese werden zwar in der Regel unter der - sofortigen - i.v.-Glukose-Gabe rasch wach werden und einen normalen oder auch überhöhten Blutzuckerspiegel erreichen. In der Folgezeit können sie aber bis zu 72 Stunden erneut Unterzuckerungsreaktionen erleiden, da die Beta-Zellen der Bauchspeicheldrüse zufolge der Sulfonylharnstoff-Gabe über längere Zeit für Glukose "sensibilisiert" bleiben. Auf einen erneuten GlukoseStimulus können diese - auch unter laufender Glukose-Infusion zufolge verstärkter Insulin-Ausschüttung erneut in einen Unterzuckerungszustand geraten. Darauf weisen in diesem Heft die Autoren des Beitrags über hypoglykämische Notfälle eindrücklich hin (vgl. auch Pfohl und Ehren, 2002).

Medikamenten-induzierte Hypoglykämien treten grundsätzlich nur bei Gabe von insulotropen oralen Antidiabetika auf, also bei Sulfonylharnstoffen oder, wenn auch wesentlich seltener, bei den Gliniden Repaglinid und Nateglinid. Am häufigsten werden sie unter Insulinbehandlung registriert. Wenn diese Substanzen zu einer Monotherapie mit nicht-insulotropen oralen Antidiabe-

\section{Hypoglykämie - eine wichtige Differential- diagnose bei jedem bewusstlosen Patienten}

tika wie Biguaniden (Metformin), Alphaglukosidasehemmern (Acarbose, Miglitol) oder Glitazonen (Rosiglitazon, Pioglitazon) dazugegeben werden, welche allein zu keiner Unterzuckerungsreaktion führen, können ebenfalls Hypoglykämien auftreten.

Bei Vorliegen einer Hypoglykämie gilt noch ein weiterer Grundsatz: Es muss immer an die Möglichkeit einer ArzneimittelInteraktion gedacht werden. Wenn diese auch recht selten sind, so gibt es doch pharmakokinetische Interaktionen, wobei Stoffe mit nahezu vollständiger Metabolisierung in der Leber oder Exkretion durch die Nieren oder auch mit hoher Plasma-Protein-Bindung suspekt sind. Hier sind beispielhaft Phenprocoumon (Marcumar) oder Bezafibrat (Cedur) anzuführen. Auch Salizylate können die renale Ausscheidung von Sulfonylharnstoffen hemmen. Andererseits gibt es die pharmakodynamischen Interaktionen, wobei diejenigen Stoffe suspekt sind, die ihrerseits den Blutzucker-Spiegel absenken können wie z.B. Clonidin (Catapresan). Alkohol, insbesondere eine akute Alkoholintoxikation, kann zu einer Hypoglykämie führen. Die blutzuckersenkende Wirkung aller Antidiabetika wird durch Alkohol auch verstärkt. Solche Hypoglykämien sind besonders gefährlich, da durch Alkohol die Glukoneogenese gehemmt wird und es manchmal zum fatalen Ausgang kommt. An sich sind, sieht man von der Alkoholintoxikation ab, ArzneimittelAktionen erfreulicherweise sehr selten Ursache einer schweren Hypoglykämie. Dennoch sollte man bei Hypoglykämien immer an die Möglichkeit pharmakokinetischer oder pharmakodynamischer Wechselwirkungen denken (Ammon, 2002).

Fazit: Der Notarzt muss bei jedem Bewusstseinsverlust oder Verwirrtheitszustand eine Hypoglykämie als Ursache differentialdiagnostisch in Erwägung ziehen und den Blutzuckerspiegel sofort mit einem Stix testen.

\section{Literatur}

1. Ammon HPT. Wechselwirkungen von Antidiabetika mit anderen Arzneistoffen. In: Schatz H (Hrsg.). Diabetologie kompakt. Berlin-Wien: Blackwell, 2002: 346-349 2. Holstein A, Plaschke A, Hammer C, Egberts E-H. Characteristics and time course of severe glimepiride - versus glibenclamide-induced hypoglycaemia. Europ J Clin Pharmacology 2003, im Druck

3. Pfohl M, Ehren M. Hypoglykämie. In: Schatz H (Hrsg.). Diabetologie kompakt. Berlin-Wien: Blackwell, 2002: 316-322

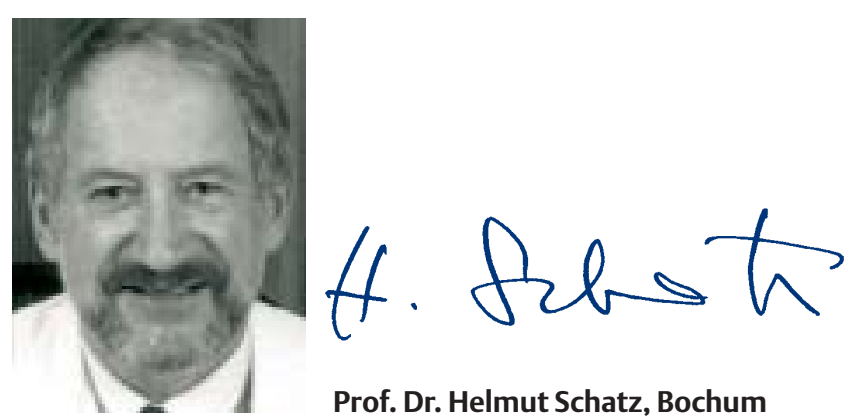

